\title{
Short-term Complications Have More Effect on Cost-effectiveness of THA than Implant Longevity
}

\author{
David W. Shearer MD, MPH, Jiwon Youm MD, MS, \\ Kevin J. Bozic MD, MBA
}

Received: 7 July 2014 / Accepted: 10 December 2014 / Published online: 6 January 2015

(C) The Association of Bone and Joint Surgeons (B) 2014

\begin{abstract}
Background Outcomes research in THA has focused largely on long-term implant survivorship as a primary outcome and emphasized the development of new implant technologies. In contrast, strategies to improve short-term outcomes, such as the frequencies of periprosthetic joint infections and unplanned readmissions, have received less attention.

Questions/purposes We asked whether reductions in periprosthetic joint infections and early readmissions

One of the authors $(\mathrm{KJB})$ certifies that he has received or may receive payments or benefits, during the study period, an amount less than USD 10,000 from Institute for Healthcare Improvement (Cambridge, MA, USA), less than USD 10,000 from Harvard Business School (Cambridge, MA, USA), and USD 10,000-USD 100,000 from Pacific Business Group on Health (San Francisco, CA, USA).

All ICMJE Conflict of Interest Forms for authors and Clinical Orthopaedics and Related Research editors and board members are on file with the publication and can be viewed on request. Each author certifies that the institution where the work was performed approved the human protocol for this investigation and that all investigations were conducted in conformity with ethical principles of research.

This work was performed at the University of California, San Francisco, CA, USA.
\end{abstract}

D. W. Shearer, K. J. Bozic $(\square)$

Department of Orthopaedic Surgery, University of California,

San Francisco, 500 Parnassus Avenue, MU 320-W,

San Francisco, CA 94143, USA

e-mail: Kevin.bozic@ucsf.edu; bozick@orthosurg.ucsf.edu

J. Youm

Department of General Surgery, University of California,

San Francisco, San Francisco, CA, USA

K. J. Bozic

Philip R. Lee Institute for Health Policy Studies, University

of California, San Francisco, San Francisco, CA, USA would have greater influence on the net monetary benefit (a summation of lifetime cost and quality of life) for THA compared with equivalent reductions in aseptic loosening. Methods A Markov model was created using decisionanalysis software with six health states and death to represent seven major potential outcomes of THA. We compared the effect of a $10 \%$ reduction in each of the following outcomes: (1) periprosthetic joint infection, (2) hospital readmission, and (3) aseptic loosening. Procedure costs (not charges) were derived from our hospital costaccounting system. Probabilities were derived primarily from the Australian Orthopaedic Association National Joint Replacement Registry, and utilities were estimated from a previous study at our institution using the time trade-off method. The primary outcome of the study is the net monetary benefit, which combines the reductions in cost and improvement in health-related quality of life in a single metric. Quality of life is expressed in quality-adjusted life years (QALYs), which are calculated by multiplying the utility of a health state (ranging from 0 to 1 ) by the duration of time in the health state. The cost and QALYs are reported separately as secondary outcomes. One-way and multivariate sensitivity analyses were performed including a probabilistic sensitivity analysis to account for uncertainty in model inputs.

Results The net monetary benefit for a $10 \%$ reduction in periprosthetic joint infections was USD 278 (95\% CI, USD 239-295) per index procedure compared with USD 174 (95\% CI, USD 150-185) and USD 113 (95\% CI, USD 94 129) for reductions in aseptic loosening and early readmission, respectively. Compared with the base case, reductions in cost associated with a $10 \%$ reduction in periprosthetic joint infections, early readmissions, and aseptic loosening were USD 98, USD 93, and USD 75 per index procedure, respectively. The increase in QALYs for 
an equivalent reduction in periprosthetic joint infections, aseptic loosening, and early readmissions were 0.0036 , 0.002, and 0.0004 QALYs, respectively. Results were most sensitive to age, baseline rate of readmission, periprosthetic joint infection, aseptic loosening, and the costs of readmission and revision THA.

Conclusions Strategies to reduce periprosthetic joint infections may have a greater effect on the cost and longterm effectiveness of THA than further enhancements in implant longevity. Reductions in the rate of readmission resulted in greater reductions in cost but not quality-of-life, and therefore had smaller effect on the net monetary benefit compared with aseptic loosening. Surgeons preferentially should engage in strategies focusing on periprosthetic joint infections to improve the value of THA care.

Level of Evidence Level II, economic and decision analysis.

\section{Introduction}

Although primary THA is considered a highly cost-effective procedure for alleviating pain and improving function in patients who have disabling hip disease [8, 15], it nonetheless accounts for a substantial (and increasing) portion of US healthcare costs. The total hospital costs for hip and knee arthroplasty combined exceeded USD 19 billion in 2010 in the United States [7]. With the demand for primary THA expected to increase $174 \%$ to nearly 572,000 procedures per year by 2030 [14], efforts to contain costs are becoming increasingly important.

Historically, the most common causes of THA failure have been related to limitations in implant longevity, such as bearing surface wear, osteolysis, and aseptic prosthetic loosening $[12,13]$. Therefore, a primary focus of THA outcomes research has been on developing new implant designs to improve implant longevity. However, with the advent of improved bearing surfaces and superior methods of implant fixation, other modes of failure in many cases unrelated to implant design, such as instability and infection, have become more common causes of THA failure [5], and may play a greater role in determining the long-term cost-effectiveness of THA. Similarly, postoperative medical complications necessitating hospital readmission, such as cardiac events and venous thromboembolism, may be an important driver of rising costs [3, 18, 19, 22, 23]. We therefore sought to assess the relative effect of reductions in each category of complication on the overall cost-effectiveness of THA. Specifically, we aimed to evaluate total cost and health-related quality of life as measured in quality-adjusted life years (QALYs). To combine these two values in one estimate of cost-effectiveness, we used the net monetary benefit, which estimates the value of health benefits in a dollar value that can be combined as a sum with the pure cost savings.
Although the ideal study would directly measure the lifetime health and economic benefits of various strategies to reduce complications of THA, conducting studies that span a lifetime typically is not feasible, and more importantly, not effective for addressing questions that cannot wait decades to answer. Therefore, modeling offers an attractive alternative as it allows projection of costs and health benefits with time by making rational assumptions based on best-available evidence. Markov modeling is a specific method of projecting outcomes that simplifies a spectrum of potential health outcomes into discrete health states each associated with a finite cost and health benefit. By adding transition probabilities between each health state, the model can be cycled repeatedly until a hypothetical cohort of patients has moved through the health states during a lifetime and in the process accumulated the associated costs and health benefits. Uncertainties in the model parameters are addressed using sensitivity analysis, whereby each input is varied along a plausible range of values to assess the effect on the model output.

In this study we used a Markov model to ask whether reductions in periprosthetic joint infections and early readmissions would have greater influence on the lifetime cost and improvements in patient quality of life associated with THA than equivalent reductions in failures related to limitations in implant longevity. Our primary outcome variable was the difference in net monetary benefit comparing equivalent $(10 \%)$ relative reductions in the rate of periprosthetic joint infections, early postoperative readmission, and revision for aseptic loosening.

\section{Methods}

A Markov model was created using TreeAge Pro 2012 (TreeAge Software Inc, Williamstown, MA, USA) to represent the potential outcomes of primary THA (Fig. 1). The study was designed according to the outline provided by the Panel on Cost-effectiveness in Health and Medicine [20]. The model starts with a 70-year-old US population based on data from the Agency for Healthcare Research \& Quality [11]. There were six patient health states considered after THA (1) well-functioning THA, (2) failed THA, (3) infected THA, (4) well-functioning revision THA, (5) successful two-stage revision THA, and (6) failed twostage revision THA. A seventh state was death (Fig. 1). The inputs for the baseline scenario were derived from best available literature (Table 1). The three hypothetical interventions compared with baseline were a $10 \%$ reduction in periprosthetic joint infections requiring two-stage revision; unplanned hospital readmission postoperatively in 90 days; and revision attributable to aseptic loosening. 


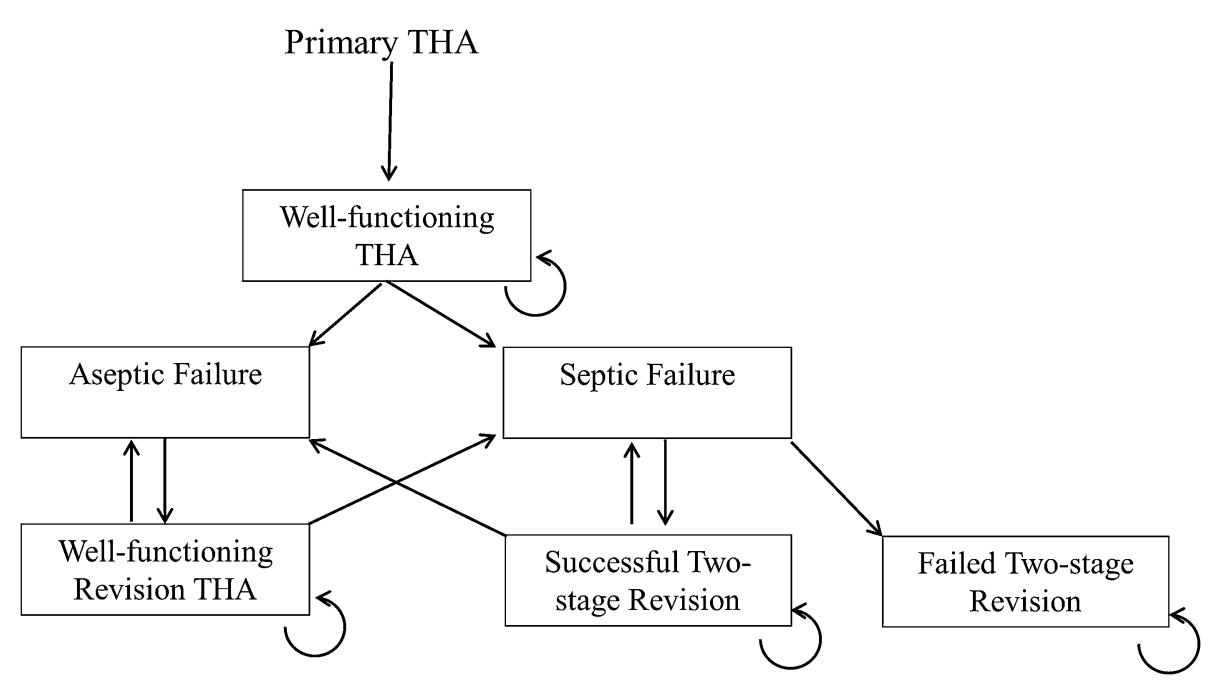

Fig. 1 The flow diagram shows the hypothetical cohort as it moves through each of the six health states with each cycle of the model. The straight arrows represent transitions to a new health state, while the

The primary outcome considered was the net monetary benefit using a willingness-to-pay threshold of USD 50,000 per QALY. The net monetary benefit is a summation of the pure monetary cost and quality of life benefit. By multiplying the quality of life benefit as measured in QALYs by the willingness-to-pay (WTP) threshold, the monetary value of an improvement in health can be estimated in units of dollars and therefore added to the change in cost. The following formula for net monetary benefit (NMB) was used:

$$
\begin{aligned}
\mathrm{NMB}= & \left(\text { Utility }_{\text {Intervention }}-\text { Utility }_{\text {Baseline }}\right) \\
& * \text { WTP }-\left(\text { Cost }_{\text {Intervention }}-\text { Cost }_{\text {Baseline }}\right)
\end{aligned}
$$

The annual probabilities of THA failure were based on the Australian Orthopaedic Association National Joint Replacement Registry [1,2]. Although we recognize that ideally there would be a systematic review for every variable in the model, especially the rate of failure, in our case we needed the best available evidence that showed long-term survivorship broken down by mode of failure and the use of highly crosslinked polyethylene, which has been shown to substantially reduce rates of revision for wear, osteolysis, and aseptic loosening. The Australian Orthopaedic Association National Joint Replacement Registry is recognized as the most valid source for this information, and also has the added benefit of generalizability because it reports results in a broader population than a focused clinical study. In addition, we were able to avoid the pitfalls of pooling data from a heterogeneous grouping of clinical studies, which can be highly problematic especially with variable duration of followup. The remaining probabilities were derived from best available primary literature (Table 1). cyclical arrows represent the cohort remaining in the same health state during the cycle. The model continues to cycle until the entire cohort has transitioned to a death state based on published life tables.

The costs of primary and revision THAs were estimated using our hospital cost-accounting system. We report costs of care based on activity-based costing rather than hospital charges. The cost of two-stage revision THA for periprosthetic joint infection was derived from a previous study which estimated costs associated with infected THA [6]. The health state utilities after successful THA, failed THA, and chronically infected THA were based on a study [4] that used the time trade-off technique to estimate utility [4]. The utility of a successful two-stage revision was estimated to be intermediate between a chronically infected THA and a successful revision THA. We were unable to identify any primary literature that addressed health utility in this population and therefore assumed that the true value would not be higher than an aseptic revision nor would it be lower than a chronically infected THA. We used a point estimate of halfway between these two values and sensitivity analysis to further explore its effect. Reoperations were associated with a disutility owing to the temporarily undesirable postsurgical state [9, 17]. A disutility was estimated for readmission, although it was $1 / 2$ the magnitude of the postsurgical disutility. Because the health impact of readmission can be highly variable depending on its cause, we elected to use a smaller, more conservative disutility than the disutility associated with undergoing a reoperation. However, we therefore specifically targeted this variable in sensitivity analysis to determine its effect on the results.

A Markov model is a type of computer simulation for use in decision analysis that divides the potential outcomes of a medical condition into discrete health states. Unlike a simple decision tree, a Markov model allows estimation of events that occur longitudinally in time by cycling the 
Table 1. Estimates for probabilities, costs, and utilities

\begin{tabular}{|c|c|c|c|c|}
\hline Variable & Estimate & Low & High & Reference(s) \\
\hline Rate of aseptic loosening (first year) & 0.002 & 0.001 & 0.003 & {$[1,2]$} \\
\hline Rate of aseptic loosening (annually*) & 0.0012 & 0.0006 & 0.0018 & {$[1,2]$} \\
\hline Rate of periprosthetic joint infection (first year) & 0.003 & 0.0015 & 0.0045 & {$[1,2]$} \\
\hline Rate of periprosthetic joint infection (annually*) & 0.0004 & 0.0002 & 0.0006 & {$[1,2]$} \\
\hline Rate of periprosthetic fracture (first year) & 0.003 & 0.0015 & 0.0045 & {$[1,2]$} \\
\hline Rate of periprosthetic fracture (annually*) & 0.0005 & 0.0003 & 0.0008 & {$[1,2]$} \\
\hline Rate of dislocation (first year) & 0.004 & 0.002 & 0.006 & {$[1,2]$} \\
\hline Rate of dislocation (annually*) & 0.0007 & 0.0004 & 0.001 & {$[1,2]$} \\
\hline Annual failure rate of revision THA & 0.046 & 0.034 & 0.058 & {$[1,2]$} \\
\hline Two-stage revision failure rate & 0.22 & 0.1 & 0.3 & [7] \\
\hline Readmission rate within 90 days & 0.089 & 0.075 & 0.109 & {$[7,8]$} \\
\hline Age at time of primary THA (years) & 70 & 55 & 85 & [11] \\
\hline Cost of primary THA (USD) & 24,200 & 18,150 & 30,250 & Institutional database \\
\hline Cost of revision THA (USD) & 34,700 & 26,000 & 43,375 & Institutional database \\
\hline Cost of two-stage revision (USD) & 96,200 & 50,000 & 150,000 & {$[4,11]$} \\
\hline Cost of readmission (USD) & 10,450 & 7000 & 13,320 & [11] \\
\hline Utility after primary THA & 0.96 & 0.94 & 0.98 & [3] \\
\hline Utility after revision THA & 0.84 & 0.71 & 0.97 & [3] \\
\hline Utility of failed THA & 0.59 & 0.46 & 0.72 & [3] \\
\hline Utility of successful two-stage revision & 0.65 & 0.46 & 0.84 & Estimated value \\
\hline Utility of failed two-stage revision (infection) & 0.46 & 0.31 & 0.61 & [3] \\
\hline Disutility of revision & -0.1 & -0.2 & 0 & {$[5,6]$} \\
\hline Disutility of two-stage revision & -0.2 & -0.3 & 0 & {$[5,6]$} \\
\hline Disutility of readmission & -0.05 & -0.1 & 0 & Estimated value \\
\hline
\end{tabular}

* Annual rate is variable based on the probabilities obtained from the Australian Orthopaedic Association National Joint Replacement Registry.

model; that is, rerunning it with changes that can occur with the passage of time. With each cycle of the model, a hypothetical cohort moves between health states using assigned probabilities. In our study, the cycle length is 1 year. Each health state is associated with a utility and cost, which are accrued for each cycle. In our study, the model continues to cycle until the entire cohort reaches the death state, thereby allowing estimation of the lifetime cost and utility of the hypothetical cohort. By varying the probabilities of specific events, such as failure of the THA, we are able to make comparisons between the proposed interventions. The specific interventions modeled were a $10 \%$ reduction in the rate of each complication, with the reference case being an approximately $0.11 \%$ annual rate ( $0.2 \%$ at 1 year, $1.5 \%$ cumulative at 12 years) of failure attributable to aseptic loosening [2]; a $0.04 \%$ annual rate of periprosthetic joint infection $(0.3 \%$ at 1 year, $0.75 \%$ cumulative at 12 years) [2]; and an $8.9 \%$ rate of 90-day unplanned readmission (range, 7.5\%-10.9\%) [23]. The model discounts the cost and quality of life associated with events that occur later to incorporate natural time preferences. Based on recommendations of the US Panel on
Cost-effectiveness in Health and Medicine, the discount rate used in our model was 3\% per year [20].

To account for uncertainty in the model parameters, oneway and multivariate sensitivity analyses were performed. As a first step, a tornado diagram was generated to determine the relative effect of each parameter independently. The most sensitive variables subsequently were tested individually using one-way sensitivity analysis and thresholds were identified that altered the preferred strategy. To account for variability on all parameters simultaneously, a probabilistic sensitivity analysis was performed. A probabilistic sensitivity analysis uses a distribution rather than point estimates for each model parameter and produces a probability of preferring each strategy rather than one preferred strategy [10].

\section{Results}

The net monetary benefit for a $10 \%$ reduction in periprosthetic joint infections was greater than that for an equivalent reduction in aseptic loosening, which in turn was greater than an equivalent reduction in early 
readmission. Reducing the rate of periprosthetic joint infections resulted in a net monetary benefit of USD 278 compared with USD 174 for aseptic loosening and USD 113 for early readmission The largest reduction in cost was associated with a $10 \%$ reduction in the rate of periprosthetic joint infections (USD 98), followed by an equivalent reduction in early postoperative readmission (USD 93). The smallest reduction in cost occurred with reduction in the rate of aseptic loosening (USD 75). With respect to quality of life, reducing the rate of periprosthetic joint infections resulted in an improvement of 0.0036 QALYs, compared with 0.002 and 0.0004 QALYs for aseptic loosening and early readmission, respectively (Fig. 2).

In one-way sensitivity analysis, the net monetary benefit was most strongly influenced by the age of the cohort and the estimated cost, utility, and baseline probability of each complication (Table 2). There was no threshold in age where reducing the rate of aseptic loosening was the preferred strategy, but with patient age greater than 88 years, reducing early readmissions resulted in higher net

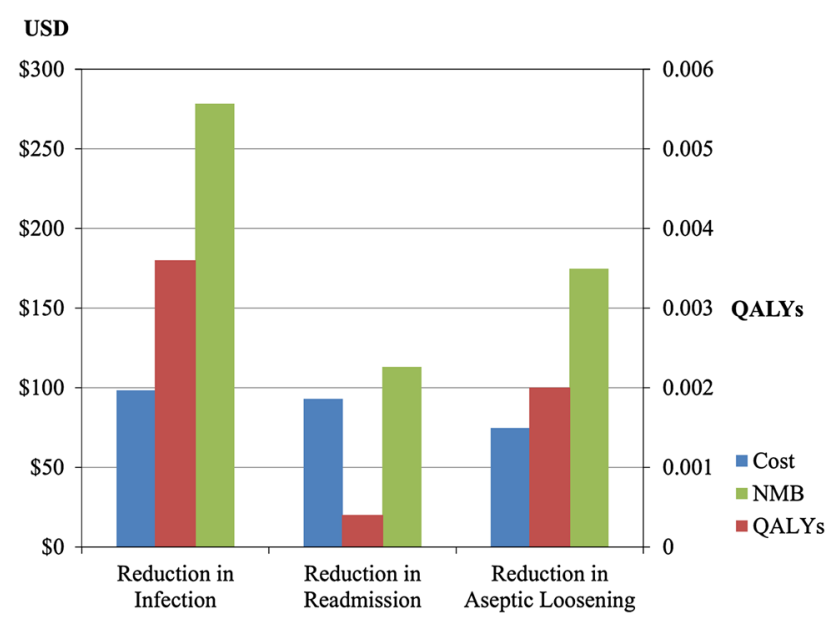

Fig. 2 Improvements in cost, quality of life, and net monetary benefit are shown in this graph. $\mathrm{NMB}=$ net monetary benefit; QALY $=$ quality-adjusted life year. monetary benefit than reducing periprosthetic joint infections. With respect to baseline rate of failure, aseptic loosening would not become the preferred strategy unless the annual rate of aseptic loosening in the base case was more than $61 \%$ higher than rates reported in the Australian Orthopaedic Association National Joint Replacement Registry for THA with a metal on highly crosslinked polyethylene bearing. Reducing rates of early postoperative readmission would become the preferred strategy if the baseline rate of readmission was greater than $21 \%$ or the cost of early postoperative readmission was greater than USD 28,900, which is shown graphically in a two-way sensitivity analysis (Fig. 3).

In the probabilistic sensitivity analysis, reducing periprosthetic joint infections was the preferred strategy in more than $99 \%$ of simulations, with a $95 \%$ CI for the net monetary benefit ranging from USD 239 to USD 295. In contrast, the $95 \% \mathrm{CI}$ for the net monetary benefit for reducing early

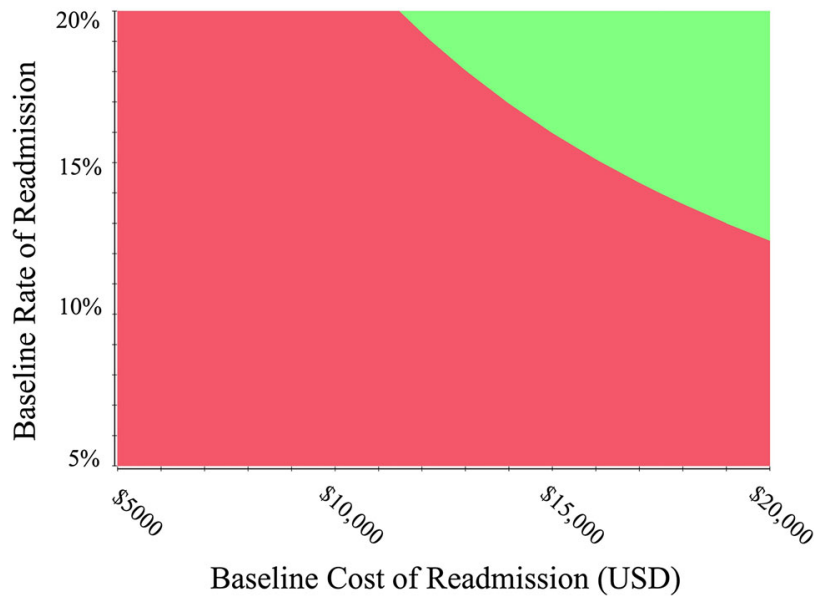

Fig. 3 A two-way sensitivity analysis was done to evaluate the effect of uncertainty in the cost of readmission and the probability of readmission on the decision to reduce readmissions or infections. The green area denotes a preference to reduce readmission, and the red area indicates a preference to reduce infection.

Table 2. Thresholds identified in the optimal strategy for net monetary benefit using one-way sensitivity analysis

\begin{tabular}{llll}
\hline Factor & Readmission $>$ infection & Loosening $>$ infection & Readmission $>$ loosening \\
\hline Age (years) & $>88$ & Robust* & $>79$ \\
Utility of revision THA & No effect & $<0.69$ & Robust \\
Utility after successful two-stage revision & Robust & Robust & No effect \\
Disutility of readmission & $>0.42$ & No effect & $>0.18$ \\
Cost of two-stage revision & Robust & Robust & No effect \\
Cost of readmission & $>$ USD 28,900 & No effect & $>$ USD 17,000 \\
Cost of revision for aseptic loosening & Robust & Robust & Robust \\
Annual rate of aseptic loosening & Robust & $>61 \%$ relative risk & $<35 \%$ relative risk \\
Probability of 90 -day readmission & $>21.6 \%$ & No effect & $>13.5 \%$ \\
\hline
\end{tabular}

* Robust defined as no identifiable threshold in plausible range of values. 
postoperative readmission was USD 94 to USD 129, and for aseptic loosening USD 150 to USD 185. The willingnessto-pay threshold did not significantly affect the results.

\section{Discussion}

Historically, a primary focus of THA outcomes research has been focused on improving implant survivorship. However, with the advent of highly crosslinked polyethylene and lower rates of wear, osteolysis, and aseptic loosening, the relative importance of more short-term outcomes, such as periprosthetic joint infections and hospital readmissions, may have increased. We used predictive modeling to evaluate the effect of reducing short- and long-term complications of THA on lifetime cost and quality of life. The model suggests that reductions in the rate of periprosthetic joint infections may result in greater improvements in cost and quality of life compared with equivalent reductions in the rate of aseptic loosening, while reducing early postoperative readmissions had greater effect on cost. However, the savings associated with reductions in the rate of readmissions were more than offset by the greater improvements in quality of life associated with reductions in aseptic loosening as reflected in the net monetary benefit. There are several logical explanations for these results. First, although periprosthetic joint infections are not the most common complication after THA, they are associated with high monetary cost and a greater reduction in quality of life when compared with revisions for aseptic loosening. In addition, there is a natural time preference for early benefits over delayed benefits, known as discounting. The same phenomenon leads to a preference for late rather than early complications. The negative effect of early complications therefore is weighted more heavily in the model.

Our study has several notable limitations. As a modelbased cost-effectiveness study, the accuracy of the results is limited by the accuracy of the model inputs. Because of the specific data points that were sought, we did not perform a systematic review for every variable. Nonetheless, we have attempted to assemble the best available literature to inform the model and used sensitivity analysis to account for uncertainty. Furthermore, despite a lack of research, decisions must be made regarding investment of research and development resources to reduce costs and improve patient quality of life after THA. As such, we would advocate for a quantitative, transparent approach to such decisions over a less systematic approach. Certain assumptions required extrapolation from the existing literature, such as the utility associated with successful two-stage revision THA for periprosthetic joint infections. However, there was no change in the preferred strategy (reduction in the rate of periprosthetic joint infections), even if the utility associated with successful aseptic and septic revisions was assumed to be equal during the sensitivity analysis. Similarly, we conservatively estimated the utility of readmission to be $1 / 2$ of a primary operation, which may underestimate the health effect of a readmission. Our costs were obtained from our hospital cost-accounting system, which estimates direct costs of care using time-driven activity-based costing. As with any hospital cost accounting system, there are limits to how direct and indirect medical costs are measured, which affects the cost estimates used in our analysis. Furthermore, there may be a lack of generalizability in our cost estimates owing to regional variation. In addition, indirect nonmedical costs, such as missed work, travel time, and other societal costs are not captured.

We are not aware of other studies that have addressed research prioritization based on various strategies to improve the cost-effectiveness of THA for direct comparison with our study. However, several studies have highlighted the substantial costs associated with early complications and readmissions after surgery [16, 21]. Using the American College of Surgeons National Surgical Quality Improvement Program linked to Medicare claims, Lawson et al. [16] reported that reducing postoperative complications for the 20 most common surgical procedures by $5 \%$ would result in savings to Medicare of USD 31 million per year. In orthopaedic surgery. Whitehouse et al. [21] estimated that surgical site infections increased the overall costs of orthopaedic procedures threefold and doubled the rate of readmissions. Although these studies are difficult to directly compare with our investigation, they support the notion that early postoperative complications have a large effect on total healthcare costs.

By design, our investigation compared the effect of equivalent reductions of three common complications of THA rather than comparing specific strategies for reducing complication rates. Our results are meant to inform decisionmaking regarding resource allocation for quality improvement efforts. We believe our results support more emphasis on reducing short-term complications, such as periprosthetic joint infections and to a lesser degree, addressing the potential cost-savings associated with reductions in early postoperative readmission. Nonetheless, our model assumed all strategies result in a proportional reduction in the rate of failure, which may not be achievable. Furthermore, the investment necessary and effort needed to achieve equivalent reductions in each complication may be vastly different. As such, the results should be interpreted cautiously and only as a rough guideline of the potential return on investment when considering research prioritization in THA. Perhaps the most important implication of our study is the implied benefit of a joint replacement registry. Registries would allow for real-time monitoring of the complications that we have shown are likely to have the greatest overall effect on 
patient health and healthcare costs associated with THA. Although long-term studies evaluating differences in implant longevity always will have value, the importance of small differences in early postoperative complications cannot be underestimated.

We have developed a cost-effectiveness model that suggests that there may be greater improvements in quality of life and cost savings associated with THA with reductions in the rate of periprosthetic joint infections compared with equivalent reductions in the rate of aseptic loosening associated with improvements in implant longevity. In addition, although the net monetary benefit resulting from reductions in the rate of aseptic loosening is larger than an equivalent reduction in the rate of hospital readmission, the pure cost savings may be greater for reductions in readmission. These findings support the notion that greater investment in strategies to improve short-term outcomes, such as reducing periprosthetic joint infection rates, rather than efforts to enhance longterm implant survivorship may be warranted.

\section{References}

1. AOA. Australian Orthopaedic Association National Joint Replacement Registry. Annual Report. Adelaide: AOA. 2009. Available at: https://aoanjrr.dmac.adelaide.edu.au/documents/10180/42728/ Annual\%20Report\%202009? version=1.1\&t=1349406243327. Accessed October 20, 2013.

2. AOA. Australian Orthopaedic Association National Joint Replacement Registry. Annual Report. 2013. Available at: https:// aoanjrr.dmac.adelaide.edu.au/en/annual-reports-2013. Accessed October 20, 2013.

3. Birkmeyer JD, Gust C, Dimick JB, Birkmeyer NJ, Skinner JS. Hospital quality and the cost of inpatient surgery in the United States. Ann Surg. 2012;255:1-5.

4. Bozic KJ, Chiu VW, Slover JD, Immerman I, Kahn JG. Health state utility in patients with osteoarthritis of the hip and total hip arthroplasty. J Arthroplasty. 2011;26:129-132 e121-122.

5. Bozic KJ, Kurtz SM, Lau E, Ong K, Vail TP, Berry DJ. The epidemiology of revision total hip arthroplasty in the United States. J Bone Joint Surg Am. 2009;91:128-133.

6. Bozic KJ, Ries MD. The impact of infection after total hip arthroplasty on hospital and surgeon resource utilization. $J$ Bone Joint Surg Am. 2005;87:1746-1751.

7. CDC Centers for Disease Control and Prevention. National Center for Health Statistics US. Osteoarthritis. Available at: http://www.cdc.gov/arthritis/basics/osteoarthritis.htm. Date Accessed June 7, 2012.
8. Chang RW, Pellisier JM, Hazen GB. A cost-effectiveness analysis of total hip arthroplasty for osteoarthritis of the hip. JAMA. 1996;275:858-865.

9. Cummins JS, Tomek IM, Kantor SR, Furnes O, Engesaeter LB, Finlayson SR. Cost-effectiveness of antibiotic-impregnated bone cement used in primary total hip arthroplasty. J Bone Joint Surg Am. 2009;91:634-641.

10. Drummond M. Methods for the Economic Evaluation of Health Care Programmes. Oxford; UK: Oxford University Press; 2005.

11. HCUP Databases. Healthcare Cost and Utilization Project (HCUP). November 2014. Agency for Healthcare Research and Quality, Rockville, MD. Available at: www.hcup-us.ahrq.gov/ databases.jsp. Accessed November 18, 2013.

12. Herberts P, Malchau H. How outcome studies have changed total hip arthroplasty practices in Sweden. Clin Orthop Relat Res. 1997;344:44-60.

13. Hirakawa K, Jacobs JJ, Urban R, Saito T. Mechanisms of failure of total hip replacements: lessons learned from retrieval studies. Clin Orthop Relat Res. 2004;420:10-17.

14. Kurtz S, Ong K, Lau E, Mowat F, Halpern M. Projections of primary and revision hip and knee arthroplasty in the United States from 2005 to 2030. J Bone Joint Surg Am. 2007;89:780-785.

15. Lavernia CJ, Alcerro JC. Quality of life and cost-effectiveness 1 year after total hip arthroplasty. J Arthroplasty. 2011;26:705-709.

16. Lawson EH, Hall BL, Louie R, Ettner SL, Zingmond DS, Han L, Rapp M, Ko CY. Association between occurrence of a postoperative complication and readmission: implications for quality improvement and cost savings. Ann Surg. 2013;258:10-18.

17. Sharifi E, Sharifi H, Morshed S, Bozic K, Diab M. Cost-effectiveness analysis of periacetabular osteotomy. J Bone Joint Surg Am. 2008;90:1447-1456.

18. Silber JH, Rosenbaum PR, Kelz RR, Reinke CE, Neuman MD, Ross RN, Even-Shoshan O, David G, Saynisch PA, Kyle FA, Bratzler DW, Fleisher LA. Medical and financial risks associated with surgery in the elderly obese. Ann Surg. 2012;256:79-86.

19. Vorhies JS, Wang Y, Herndon J, Maloney WJ, Huddleston JI. Readmission and length of stay after total hip arthroplasty in a national Medicare sample. J Arthroplasty. 2011;26:119-123.

20. Weinstein MC, Siegel JE, Gold MR, Kamlet MS, Russell LB. Recommendations of the Panel on Cost-effectiveness in Health and Medicine. JAMA. 1996;276:1253-1258.

21. Whitehouse JD, Friedman ND, Kirkland KB, Richardson WJ, Sexton DJ. The impact of surgical-site infections following orthopedic surgery at a community hospital and a university hospital: adverse quality of life, excess length of stay, and extra cost. Infect Control Hosp Epidemiol. 2002;23:183-189.

22. Wolf BR, Lu X, Li Y, Callaghan JJ, Cram P. Adverse outcomes in hip arthroplasty: long-term trends. J Bone Joint Surg Am. 2012;94:e103.

23. Zhan C, Kaczmarek R, Loyo-Berrios N, Sangl J, Bright RA. Incidence and short-term outcomes of primary and revision hip replacement in the United States. J Bone Joint Surg Am. 2007; 89:526-533. 